\title{
Effect of Ethanol Extract of Venenum Bufonis on Biofilm Formation of Staphylococcus
}

\section{aureus}

\author{
Bing Yan', Man Guo', Liping Zhao ${ }^{2 *}$ \\ ${ }^{1}$ School of Life Sciences and Technical, Mudanjiang Normal University, Mudanjiang, China \\ ${ }^{2}$ School of Life Sciences, University of Science and Technology of China, Hefei, China \\ Email: zhaolp@ustc.edu.cn
}

Received 29 August 2014; revised 13 October 2014; accepted 2 November 2014

Copyright (C) 2014 by authors and Scientific Research Publishing Inc.

This work is licensed under the Creative Commons Attribution International License (CC BY). http://creativecommons.org/licenses/by/4.0/

(c) (i) Open Access

\begin{abstract}
Ethanol extract of Venenum Bufonis has shown many valuable bioactivities, but little is known about its effect on biofilm formation of Staphylococcus aureus. In this study, biofilm formation of $S$. aureus NCTC8325 with or without ethanol extract of Venenum Bufonis was tested using microtitre plate assay and Confocal Laser Scanning Microscope (CLSM) system. Results show that the biofilm formation of $S$. aureus with ethanol extract of Venenum Bufonis was significantly lower than that of the control group without ethanol extract of Venenum Bufonis. Meanwhile, the reduction degree was correlated to the concentration of ethanol extract of Venenum Bufonis positively. With CLSM system we can observe that looser and less biomass of the biofilm structure of the experimental group appeared than that of the control group. These results suggested that ethanol extract of Venenum Bufonis has inhibitory effect on biofilm formation of $S$. aureus.
\end{abstract}

\section{Keywords}

Ethanol Extract of Venenum Bufonis, S. aureus, Biofilm Formation

\section{Introduction}

Staphylococcus aureus, as an important opportunistic pathogen is a leading cause of human bacterial infection worldwide and is endemic in both hospital and community, which can cause potentially life-threatening nosocomial and community-acquired infections, such as osteomyelitis and endocarditis [1] [2]. S. aureus has the ability to adhere to many tissues and implants in humans to form biofilms, which enables it to survive in antimicrobial stress and immune responses causing refractory chronic infections. Novel strategies that target biofilm-

\footnotetext{
*Corresponding author.
} 
associated infections caused by S. aureus will be considered as future therapies [3]-[5].

Venenum Bufonis, a traditional Chinese medicine used as a topical anesthetic and cardiac medication, is extracted from the dried white secretion of auricular glands and skin glands of Chinese toad (Bufo melanostictus Schneider or Bufo bufo gargarzinas Gantor). Ethanol extract of Venenum Bufonis has shown strong pharmacological activity, including the effects on antiproliferative [6] and on anti-osteosarcoma [7], killing various cancer cells by inducing their apoptosis [8] [9]. However, to date, the effect of ethanol extract of Venenum Bufonis on $S$. aureus biofilm formation has not been reported. In this study, we investigated the effects of ethanol extract of Venenum Bufonis against biofilm formation of $S$. aureus in vitro, and concluded that ethanol extract of Venenum Bufonis has inhibitory effect on biofilm formation of S. aureus.

\section{Materials and Methods}

\subsection{Bacterial Strains and Growth Conditions}

The bacterial strains used in this study including S. aureus NCTC8325, and S. aureus NCTC8325pGFP carrying the fluorescent protein were provided by University of Science \& Technology China. They were grown at $37^{\circ} \mathrm{C}$ in tryptic soy broth (TSB) medium (BD) or TSB with chloramphenicol $(15 \mu \mathrm{g} / \mathrm{mL})$. Medium was supplemented with $3 \%$ sodium chloride or $0.5 \%$ glucose when necessary.

\subsection{Production of Ethanol Extracts of Venenum Bufonis}

Venenum Bufonis was purchased in China toad Venenum Bufonis industry. It was ground into powder and was suspended in $10 \mathrm{~mL} 80 \%$ ethanol solution each $0.5 \mathrm{~g}$ and extracted two times at $70^{\circ} \mathrm{C}$ water bath for $90 \mathrm{~min}$. The solvent of the extract was removed in a rotary evaporator under vacuum. The resulting residue was dissolved in $80 \%$ ethanol at $50 \mathrm{mg} / \mathrm{mL}$ extract concentration and was diluted with $80 \%$ ethanol to $25 \mathrm{mg} / \mathrm{mL}, 12.5 \mathrm{mg} / \mathrm{mL}$, $6.25 \mathrm{mg} / \mathrm{mL}$, and $3.125 \mathrm{mg} / \mathrm{mL}$ concentration.

\subsection{Antimicrobial Activity}

Overnight cultures of S. aureus NCTC8325 were initiated by touching a sterile wire loop to three individual colonies on every plate and inoculating into $2 \mathrm{~mL}$ of TSB medium. The inoculum was cultured to a final concentration of $10^{6} \mathrm{CFU} / \mathrm{mL}$ in fresh TSB and was dispensed into 96-well plates (Corning, Steuben, NY) containing serial dilutions of ethanol extracts of Venenum Bufonis $(2.5 \mathrm{mg} / \mathrm{mL}, 1.25 \mathrm{mg} / \mathrm{mL}, 0.625 \mathrm{mg} / \mathrm{mL}, 0.3125 \mathrm{mg} / \mathrm{mL}$, $0 \mathrm{mg} / \mathrm{mL}$ ). At the same time $1 \mu \mathrm{g} / \mathrm{mL}$ vancomycin was used as standard antibiotic control. Plates were incubated at $37^{\circ} \mathrm{C}$ for $12 \mathrm{~h}$ and then ten-fold serial dilutions of cultures were performed by successive transfer $(0.1 \mathrm{~mL})$ through seven microfuge tubes containing $0.9 \mathrm{~mL}$ of TSB. Four appropriate dilutions were dropped on LB agar plates and viable colonies were counted after incubation at $37^{\circ} \mathrm{C}$ for $24 \mathrm{~h}$. Experiments were repeated three times with four parallels.

\subsection{Biofilm Formation in Microtitre Plate Assay}

Biofilm formation under static conditions was determined by the microtitre plate assay based on the method described previously [10]. Briefly, the overnight cultures were made at a 1:100 dilution using fresh TSB containing $3 \% \mathrm{NaCl}$ or $0.5 \%$ glucose. The diluted cell suspension was inoculated into flat-bottom 96-well polystyrene plates (Costar 3599, Corning Inc., Corning, NY), $200 \mu$ l each well containing serial dilutions of ethanol extract of Venenum Bufonis ( $2.5 \mathrm{mg} / \mathrm{mL}, 1.25 \mathrm{mg} / \mathrm{mL}, 0.625 \mathrm{mg} / \mathrm{mL}, 0.3125 \mathrm{mg} / \mathrm{mL}, 0 \mathrm{mg} / \mathrm{mL}$ ). The plates were incubated at $37^{\circ} \mathrm{C}$ for $12 \mathrm{~h}$ and wells were rinsed gently with water five times to remove non-adherent cells. Subsequently, the plates were stained with $0.5 \%$ crystal violet for $15 \mathrm{~min}$, and then rinsed again with water to remove unbound stain. After that, the plates were dried, and the optical density at $560 \mathrm{~nm}$ (OD560) was determined with an enzyme-linked immunosorbent assay reader (biotek Elx800, USA) in a $5 \times 5$ scan model.

\subsection{Observation of Biofilm Formation in Laser Scanning Confocal Microscope System (CLSM)}

Overnight cultures of S. aureus NCTC8325pGFP, which was transformed with the GFP plasmid for fluorescence detection from S. aureus NCTC8325, was inoculated at a 1:100 dilution in fresh TSB containing 3\% $\mathrm{NaCl}$ 
and $15 \mu \mathrm{g} / \mathrm{mL}$ chloramphenicol to maintain plasmid selection into glass bottom culture dishes ( $35 \mathrm{~mm}$ petri dish, $10 \mathrm{~mm}$ microcell, MatTek, USA), $1 \mathrm{~mL}$ each dish. The experimental group contained $1.25 \mathrm{mg} / \mathrm{mL}$ ethanol extracts of Venenum Bufonis. Dishes were incubated at $37^{\circ} \mathrm{C}$ for $12 \mathrm{~h}$ and were rinsed gently with water five times to remove non-adherent cells. CLSM was performed on a Zeiss LSM710 collected at $1 \mu \mathrm{m}$ intervals. Selected confocal images stood for similar areas of interest and each confocal experiment was repeated four times. The confocal images were acquired from Zeiss ZEN 2010 software package (Carl Zeiss, Jena, Germany) and the three-dimensional biofilm images were rendered with Imaris 7.0 (Bitplane, Zurich, Switzerland).

\subsection{Statistical Analysis}

The paired $t$ test was used for statistical comparisons between groups. The level of statistical significance was set at a $P$ value of $\leq 0.05$.

\section{Results and Discussion}

\subsection{Inhibitory Effect of Ethanol Extract of Venenum Bufonis on Sa aureus}

Figure 1 shows the viable counts of S. aureus NCTC8325 after exposure to TSB with or without the extract from Venenum Bufonis for $12 \mathrm{~h}$ at $37^{\circ} \mathrm{C}$. Compared with the control group without the extract of Venenum $\mathrm{Bu}-$ fonis, the viable counts of bacteria of the experimental group containing ethanol extract of Venenum Bufonis were decreased significantly, and the reducing extent was positively correlated with the concentration of ethanol extract of Venenum Bufonis. Viable counts were almost 100 times lower in the presence of $0.3125 \mathrm{mg} / \mathrm{mL}$ ethanol extract of Venenum Bufonis, 1000 times lower in the presence of $0.625 \mathrm{mg} / \mathrm{mL}$ ethanol extract of Venenum Bufonis and 10,000 times lower in the presence of $2.5 \mathrm{mg} / \mathrm{mL}$ ethanol extract of Venenum Bufonis compared with the control group. These data show that certain concentrations of ethanol extract of Venenum Bufonis can inhibit the growth of $S$. aureus, but the increase of ethanol extract of Venenum Bufonis concentration cannot completely kill the bacteria. However, there were no viable bacteria in the standard antibiotic control that contains $1 \mu \mathrm{g} / \mathrm{mL}$ vancomycin (data not shown).

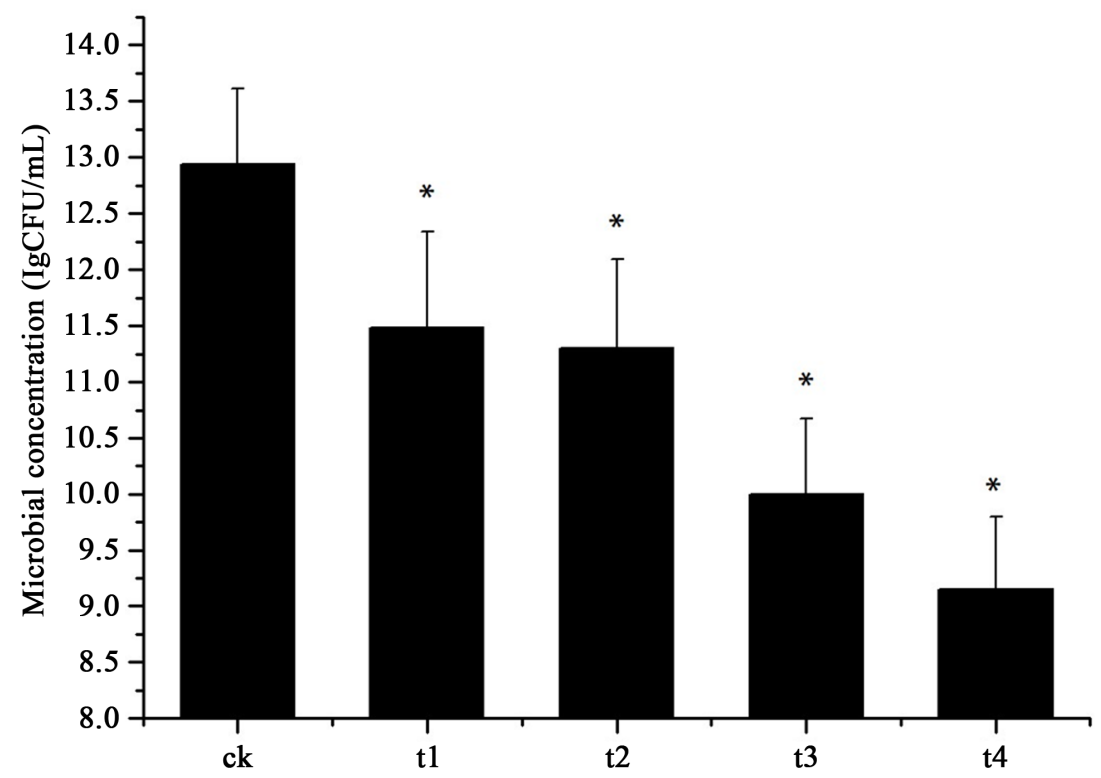

Figure 1. Colony counts (lgCFU/mL) of S. aureus NCTC8325 following 12 h of incubation at $37^{\circ} \mathrm{C}$ with or without ethanol extract of Venenum Bufonis. ck: control group no ethanol extract of Venenum Bufonis, t1: $0.3125 \mathrm{mg} / \mathrm{mL}$ ethanol extract of Venenum Bufonis, t2: $0.625 \mathrm{mg} / \mathrm{mL}$ ethanol extract of Venenum Bufonis, t3: 1.25 $\mathrm{mg} / \mathrm{mL}$ ethanol extract of Venenum Bufonis, t4: $2.5 \mathrm{mg} / \mathrm{mL}$ ethanol extract of Venenum Bufonis. *Significantly different from no Venenum Bufonis control $(\mathrm{P}<$ $0.05)$. 


\subsection{Inhibitory Effect of Ethanol Extract of Venenum Bufonis on Biofilm Formation of $S$, aureus}

To investigate whether ethanol extract of Venenum Bufonis affects biofilm formation of S. aureus, we monitored the biofilm formation of S. aureus NCTC8325 using a microtitre plate assay. As shown in Figure 2, strains of the control group without ethanol extract of Venenum Bufonis formed strong biofilm after $12 \mathrm{~h}$ incubation at $37^{\circ} \mathrm{C}$. However, strains of the experimental groups with different concentrations of ethanol extract of Venenum Bufonis formed weaker biofilm as measured by quantitative spectrophotometric analysis based on OD560 after crystal violet staining. Moreover, this discrepancy was increasing with the increase of ethanol extract of Venenum Bufonis concentration when cultivated in TSB containing 3\% NaCl (Figure 2(a)). When cultivated in TSB containing $0.5 \%$ glucose, stains of $S$. aureus with $0.625 \mathrm{mg} / \mathrm{mL}$ ethanol extract of Venenum Bufonis formed biofilm significantly more weakly than the control group (Figure 2(b)).

In order to directly observe the effect of ethanol extract of Venenum Bufonis on biofilm formation of S. $a u-$ reus, we used CLSM system and strains that can constitutively express green fluorescent protein GFP. After 12 $\mathrm{h}$ incubation at $37^{\circ} \mathrm{C}$ in TSB containing 3\% NaCL, strains of the control group without ethanol extract of Venenum Bufonis produced intact and rough biofilms as monitored by CLSM. In contrast, biofilms produced by strains of the experimental group with $1.25 \mathrm{mg} / \mathrm{mL}$ ethanol extract of Venenum Bufonis were undetectable (Figure 3).

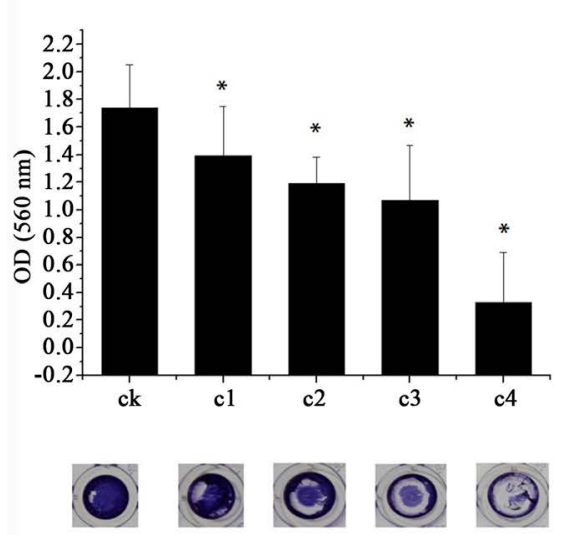

(a)

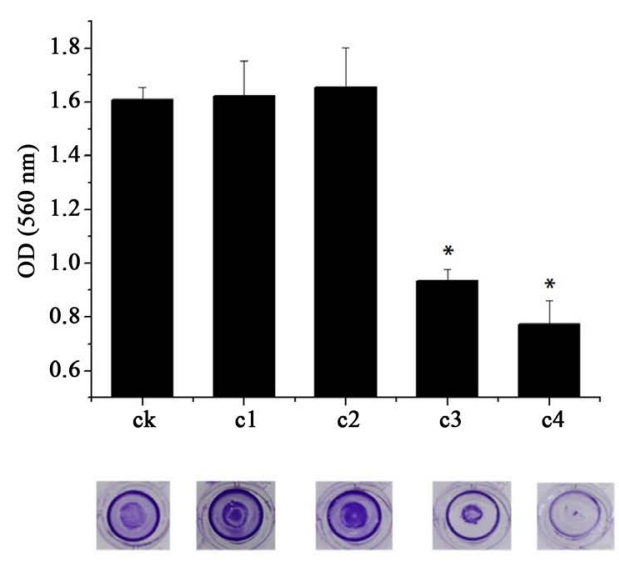

(b)

Figure 2. Biofilm formation of S. aureus NCTC8325 when cultivated in TSB containing 3\% NaCL (a) or TSB containing $0.5 \%$ glucose (b) in 96 -well plates for $12 \mathrm{~h}$ at $37^{\circ} \mathrm{C}$ with or without ethanol extract of Venenum Bufonis. The cells that adhered to the plate after staining with crystal violet were measured by OD560. ck: control group no ethanol extract of Venenum Bufonis, c1: $0.15625 \mathrm{mg} / \mathrm{mL}$ ethanol extract of Venenum Bufonis, c2: $0.3125 \mathrm{mg} / \mathrm{mL}$ ethanol extract of Venenum Bufonis, c3: $0.625 \mathrm{mg} / \mathrm{mL}$ ethanol extract of Venenum Bufonis, c4: $1.25 \mathrm{mg} / \mathrm{mL}$ ethanol extract of Venenum Bufonis. * Significantly different from no Venenum Bufonis control $(\mathrm{P}<0.05)$.

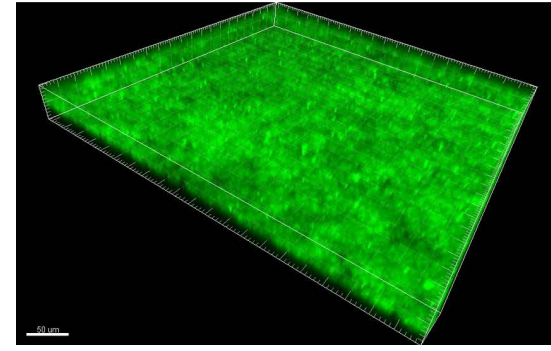

(a)

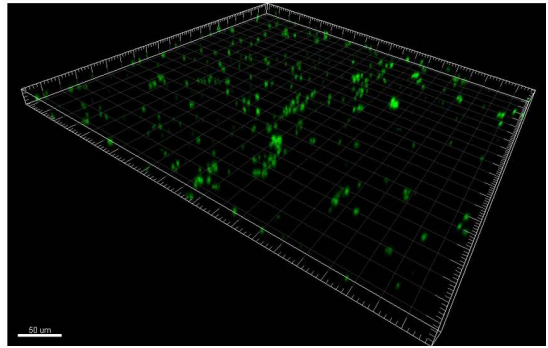

(b)

Figure 3. Observation of biofilm formation of S. aureus NCTC8325pGFP when cultivated in TSB containing $3 \% \mathrm{NaCL}$ and $15 \mu \mathrm{g} / \mathrm{mL}$ chloramphenicol in glass bottom culture dishes for $12 \mathrm{~h}$ at $37^{\circ} \mathrm{C}$ without (a) or with $1.25 \mathrm{mg} / \mathrm{mL}$ ethanol extract of Venenum Bufonis (b). Biofilm integrity and GFP fluorescence were monitored by CLSM and the three-dimensional images were rendered with Imaris 7.0. 
Biofilm formation of $S$. aureus plays an important role in catheter-related infections, which is difficult to control and brings serious threat to human health. Searching for new drugs to inhibit the biofilm formation of $S$. aureus has become a new strategy for clinical infection treatment. Here, we provide evidence that ethanol extract of Venenum Bufonis has inhibitory effect on biofilm formation of S. aureus, suggesting a potential clue for therapy in S. aureus biofilm-associated infection.

However, the biofilm formation of $S$. aureus is a complex process, which involves a variety of factors, including quorum sensing systems, two component and other signal systems from a network to regulate. This study is only a preliminary detection to the effect of ethanol extract of Venenum Bufonis on biofilm formation of $S$. aureus. More specific mechanism involved in this process remains to be further studied in detail.

On the other hand, the component of Venenum Bufonis is complex. Through separation and identification, it was proved to contain many toad toxins, bufalin ligands, bufalin tryptamines and other compounds such as morphine and epinephrine. The effective components of Venenum Bufonis in general are fat soluble, which can be extracted by $80 \%$ ethanol. The active ingredient of Venenum Bufonis that inhibits the growth and the biofilm formation of $S$. aureus can be bufadienolides and telocinobufagin; however the specific mechanism needs further study.

\section{Conclusion}

This study provided evidence that the ethanol extract of Venenum Bufonis, which is a traditional Chinese medicine, could inhibit the biofilm formation of $S$. aureus. The results suggested a potential clue for therapy in $S$. aureus biofilm-associated infection.

\section{Acknowledgements}

We thank Professor Baolin Sun in School of Life Sciences, University of Science and Technology of China for providing the strains. This work was supported by the provincial key research project of Heilongjiang Province in Mudanjiang Normal College (sy 201230).

\section{References}

[1] Lowy, F.D. (1998) Staphylococcus aureus Infections. New England Journal of Medicine, 339, 520-532. http://dx.doi.org/10.1056/NEJM199808203390806

[2] Otto, A., van Dijl, J.M., Hecker, M. and Becher, D. (2014) The Staphylococcus aureus Proteome. International Journal of Medical Microbiology, 304, 110-120. http://dx.doi.org/10.1016/j.ijmm.2013.11.007

[3] Costerton, J.W., Montanaro, L. and Arciola, C.R. (2005) Biofilm in Implant Infections: Its Production and Regulation. The International Journal of Artificial Organs, 28, 1062-1068.

[4] Del Pozo, J.L. and Patel, R. (2007) The Challenge of Treating Biofilm-Associated Bacterial Infections. Clinical Pharmacology and Therapeutics, 82, 204-209. http://dx.doi.org/10.1038/sj.clpt.6100247

[5] Archer, N.K., Mazaitis, M.J., Costerton, J.W., Leid, J.G., Powers, M.E. and Shirtliff, M.E. (2011) Staphylococcus aureus Biofilms: Properties, Regulation, and Roles in Human Disease. Virulence, 2, 445-459. http://dx.doi.org/10.4161/viru.2.5.17724

[6] Wang, S.H., Wang, Y.C., Nie, Y.L., Hai, Y.N., Sun, H.F., Yuan, Z.L. and Nan, K.J. (2013) Antiproliferative Activity of the Chinese Medicinal Compound, Delisheng, Compared with Rg3 and Gemcitabine in HepG2 Cells. Indian Journal of Pharmaceutical Sciences, 75, 578-584.

[7] Chen, G., Jiang, X., Li, J., Duan, G., Yang, L., Zhang, Y. and Wang, F. (2014). Study on Anti-Osteosarcoma Activity of Ethanol Extract of Venenum Bufonis in Vitro. African Journal of Traditional, Complementary, and Alternative Medicines. African Networks on Ethnomedicines, 11, 73-77.

[8] Zhang, D.M., Liu, J.S., Tang, M.K., Yiu, A., Cao, H.H., Jiang, L., Chan, J.Y., Tian, H.Y., Fung, K.P. and Ye, W.C. (2012) Bufotalin from Venenum Bufonis Inhibits Growth of Multidrug Resistant HepG2 Cells through G2/M Cell Cycle Arrest and Apoptosis. European Journal of Pharmacology, 692, 19-28. http://dx.doi.org/10.1016/j.ejphar.2012.06.045

[9] Li, M., Yu, X., Guo, H., Sun, L., Wang, A., Liu, Q., Wang, X. and Li, J. (2014) Bufalin Exerts Antitumor Effects by Inducing Cell Cycle Arrest and Triggering Apoptosis in Pancreatic Cancer Cells. Tumour Biology, 35, 2461-2471. http://dx.doi.org/10.1007/s13277-013-1326-6

[10] Beenken, K.E., Blevins, J.S. and Smeltzer, M.S. (2003) Mutation of sarA in Staphylococcus aureus Limits Biofilm Formation. Infection and Immunity, 71, 4206-4211. http://dx.doi.org/10.1128/IAI.71.7.4206-4211.2003 
Scientific Research Publishing (SCIRP) is one of the largest Open Access journal publishers. It is currently publishing more than 200 open access, online, peer-reviewed journals covering a wide range of academic disciplines. SCIRP serves the worldwide academic communities and contributes to the progress and application of science with its publication.

Other selected journals from SCIRP are listed as below. Submit your manuscript to us via either submit@scirp.org or Online Submission Portal.
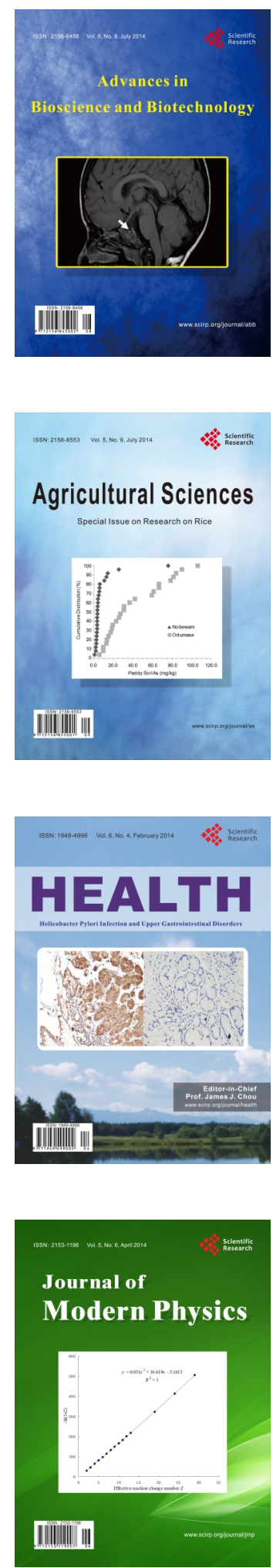
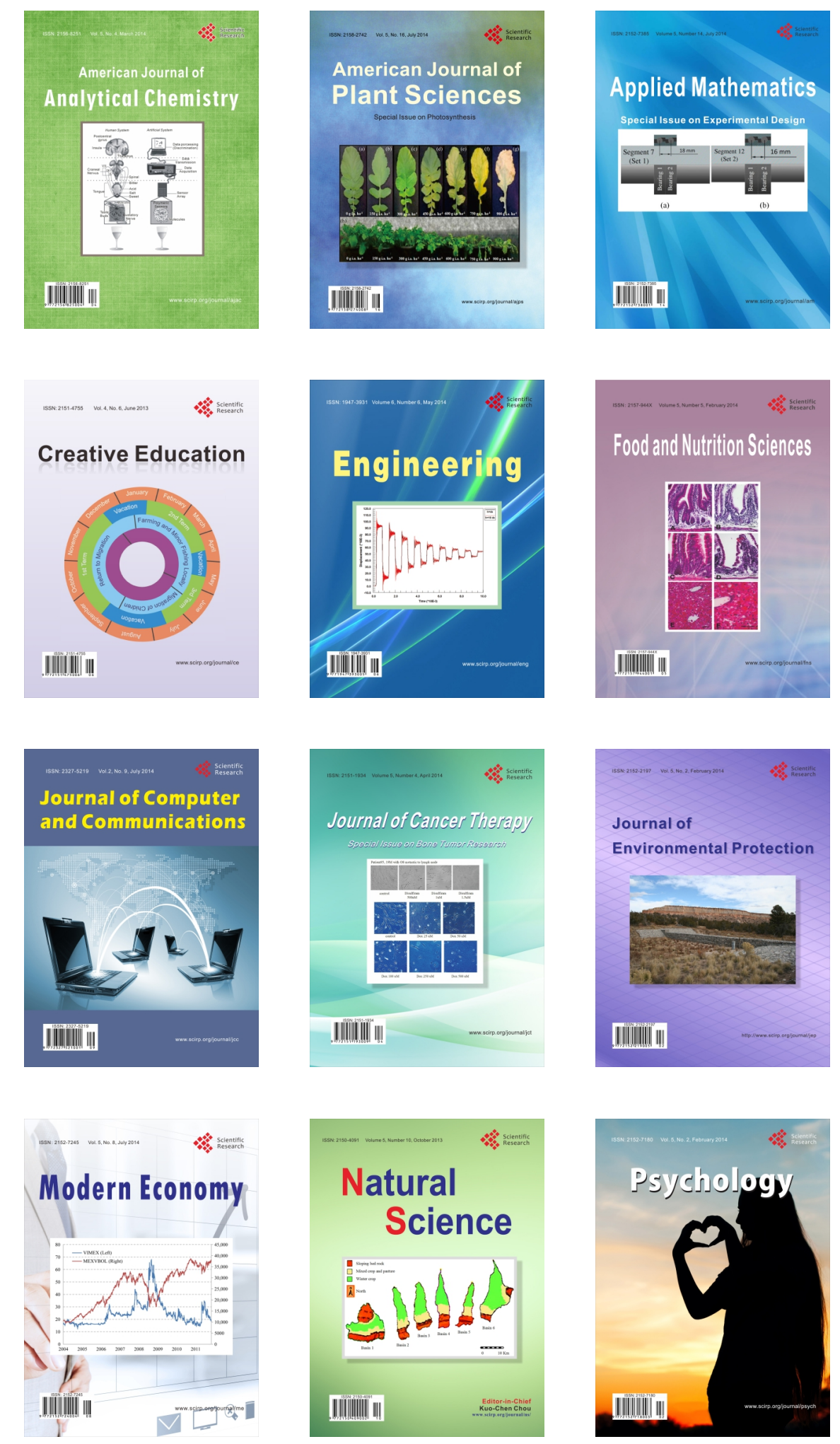